\title{
Clinical elastography
}

\section{Ioan Sporea}

Department of Gastroenterology and Hepatology, "Victor Babeș” University of Medicine and Pharmacy, Timișoara, Romania

Evolution of medicine is something normal and practitioners must be informed and prepared to accept this normal direction in life. This evolution has been so accelerating in the last 20-30 years, that sometimes we do not have the time to keep up with the new information. Hepatology is a internal medicine subspecialty, where many new things appear in a short period of time, regarding both diagnosis and therapy. The field of hepatology therapy is now very dynamic in some areas such as HCV treatment, but not only there.

Starting from Hippocrates's time, liver palpation had been for a long time the best approach to establish the severity of chronic liver disease, while 30-40 years ago, laparoscopy or liver biopsy has become the standard technique in liver evaluation. In the last 10-15 years, the development of elastography (as a new modality to palpate an organ - no matter if it is a breast nodule, the thyroid or the liver) has become the new way to evaluate the severity of the liver diseases.

Transient Elastography (FibroScan) (TE) and strain elastography have opened the way in this new modality of liver assessment, and the later, point or 2D-Shear Wave Elastographic (SWE) appeared on the market [1]. From a practical point of view, elastographic methods (TE, point SWE and 2D-SWE) assess liver stiffness as a marker of fibrosis severity [2].

The new concept of ultrasound is "point of care" (or clinical) ultrasound [3], meaning that ultrasound examination should immediately follow the clinical evaluation of an organ, in the examination room, by the specialist

Received Accepted

Med Ultrason

2018, Vol. 20, No 3, 263-264

Corresponding author: Prof. IoanSporea, MD, $\mathrm{PhD}$

13, Snagov Street,

300482 Timisoara, Romania

Phone: +40 256 309455, Fax: + 40256488003

E-mail: isporea@umft.ro in that particular field (without referring the patient to a radiologist to perform the procedure, like in the AngloSaxon/American model!). This modality has already been implemented in many countries, in specialties such as urology, obstetrics/gynecology, endocrinology, gastroenterology-hepatology and other fields. The advantages of this model are: knowledge of clinical information by the physician performing the ultrasound examination; shorter period before diagnosis; less waiting time for the patient. Despite some contradictory discussions regarding this model between Anglo-Saxon/American model and German model countries [4,5], this is probably the direction of evolution of ultrasound in the future.

Coming back to the title of the editorial: clinical elastography. What does this mean? It means to use elastography, immediately after the ultrasound evaluation, in the same room, possibly with the same equipment. This is now a standard of care for breast ultrasound, where guidelines recommend immediate stiffness evaluation if a lesion is found [6], but this is also implemented for other organs such as thyroid, liver, pancreatic EUS and others.

The use of clinical elastography in hepatology has been increasing during the last 5-10 years. In the geographical area where ultrasound is performed by hepatologists/ gastroenterologists, the clinical evaluation of a patient with liver abnormalities is followed by an ultrasound examination ("point of care" examination), that can reveal a normal ultrasound liver, a diffuse disease or a liver mass. Having an elastographic method available, the physician can perform a liver elastography during the same examination. The aim is to rule-out (to exclude) or to rule-in a severe liver disease. Despite some confounding factors (fasting or not, increased aminotransferases levels, obstructive jaundice, right heart failure and others), liver elastography is an appropriate tool for practice for the purpose that we underlined. 
Since I have been using clinical elastography for more than 10 years, I can say that this strategy changed my approach in hepatology. In all patients with potential liver diseases, after clinical and ultrasound examination (searching signs of advanced liver diseases), we perform liver elastography. For systems with elastography modules (point or 2D-SWE), the evaluation lasts less than five minutes, with $5 / 10$ valid measurements, with no discomfort for the patient. With a real-time ultrasound visualization of the liver, we can decide where to place the region of interest (ROI) for liver stiffness evaluation, avoiding the capsule or the large vessels. The accuracy of the elastographic evaluation of fibrosis severity ranges from 80 to $95 \%$, increasing with the severity of fibrosis, with good performance to rule-in or rule-out significant fibrosis.

For all the systems, there are published cut-off values for different stages of fibrosis (which unfortunately are not the same in different elastographic systems!). Usually, in daily practice there are two strategical points for the clinician: the absence of significant fibrosis (F0/F1) or the presence of severe fibrosis/cirrhosis (F3/F4), and this can be quite accurately assessed using elastography.

I believe in the development of clinical elastography, at least in the field of hepatology and this can be achieved by integrating elastographic module in middle class ultrasound equipment (and maybe, not very far, in small, portable ultrasound equipment). Since liver elastography has a short time learning curve (maybe 50/100 examinations), it can be performed by every medical specialty and it might be used in screening for liver chronic diseases (such as steato-hepatitis).

Finally, clinical elastography is an advantage in some areas. In the field of hepatology, this approach helps many clinicians to take a rapid decision, regarding prognosis and therapy. This strategy can reduce the patient's anxiety regarding disease severity and can reduce the duration of patient's evaluation.

\section{References}

1. Dietrich CF, Bamber J, Berzigotti A, et al. EFSUMB Guidelines and Recommendations on the Clinical Use of Liver Ultrasound Elastography, update 2017. Ultraschall Med 2017;38:377-394.

2. Sporea I, Bota S, Săftoiu A, et al. Romanian national guidelines and practical recommendations on liver elastography. Med Ultrason 2014;16:123-138.

3. Moore CL, Copel JA. Point-of-care ultrasonography. N Engl J Med 2011;364:2075-2076.

4. Edwards HM, Sidhu P. Who's doing your scan? A European perspective on ultrasound services. Ultraschall in Med 2017;38:479-482.

5. Seitz K. Who's Doing Your Scan? The German Perspective on Ultrasound Services: Ultrasound is More Than a Technique, it's a Medical Art. Ultraschall in Med 2017:38:661663.

6. Barr RG, Nakashima K, Amy D, et al. WFUMB guidelines and recommendation for clinical use of ultrasound elastography: Part 2: breast. Ultrasound Med Biol 2015;41:11481160. 